\title{
Crescimento em altura de diferentes híbridos de eucalipto em diferentes idades
}

Jade Ferreira Figueiredo ${ }^{\mathrm{a}}$, Maria Cristina Bueno Coelho ${ }^{\mathrm{a}^{*}}{ }^{\mathbb{0}}$, Dayane Lucena Andrade ${ }^{\mathrm{a}}$,

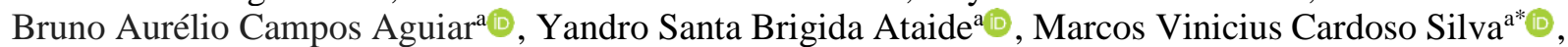
José Fernando Pereira ${ }^{a}$, Mathaus Messias Coimbra Limeira ${ }^{a}$

${ }^{a}$ Universidade Federal do Tocantins, Brasil

*Autor correspondente (mariacristina@uft.edu.br)

\section{N F O}

\section{Keywords}

hybrids

frequency distribution

normality

\begin{abstract}
A B S T R A C T
Heigth growth of diferente eucalyptus hybrids at diferente ages

The objective of this work was to evaluate the growth in total height in meters in two periods (25 and 89 months) of hybrids Eucalyptus urophylla x Eucalyptus camaldulensis (VM 58), Eucalyptus urophylla x Eucalyptus grandis (GG100) and hybrid seeds of Eucalyptus urophylla x Eucalyptus grandis. The study was carried out in the south of the state of Tocantins, in the area of the PROJECTO company in the municipality of Aliança (TO). The data were obtained from a continuous stratified inventory (per hybrid), with rectangular plots of $27.5 \times 28 \mathrm{~m}\left(770 \mathrm{~m}^{2}\right)$ each, systematically distributed in each stratum. The dendrometric variables evaluated were the CAP in $\mathrm{cm}$ (after being converted to DAP) and Total height (Ht) in $\mathrm{m}$. The results obtained based on the height gain of the trees over the two ages evaluated showed the superior performance of the GG100 clone in relation to the other genetic materials evaluated, with an average increase of $14.4 \mathrm{~m}$ in height at 25 months and $20.7 \mathrm{~m}$ in height. 89 months old. The periodic mean increment for the variables DBH $(\mathrm{cm})$ and $\mathrm{Ht}(\mathrm{m})$ was $6.65 \mathrm{~cm}$ and $8.06 \mathrm{~m}$ for hybrids Eucalyptus urophylla $\mathrm{x}$ Eucalyptus camaldulensis (VM 58); $9.4 \mathrm{~cm}$ and $6.44 \mathrm{~m}$ for hybrid of Eucalyptus urophylla $\mathrm{x}$ Eucalyptus grandis (GG100) and $9.61 \mathrm{~cm}$ and $8.36 \mathrm{~m}$ for hybrid Eucalyptus seeds. urophylla $\mathrm{x}$ Eucalyptus grandis. The hybrids of Eucalyptus urophylla x Eucalyptus grandis (GG100) and Eucalyptus seeds. urophylla $\mathrm{x}$ Eucalyptus grandis had thehighest average growth in total height $(\mathrm{m})$ and these are recommended for planting in the region.
\end{abstract}

\section{R E S U M O}

O objetivo deste trabalho foi avaliar o desenvolvimento em altura total (m) em dos híbridos Eucalyptus urophylla $\mathrm{x}$ Eucalyptus camaldulensis (VM 58), Eucalyptus urophylla $\mathrm{x}$ Eucalyptus grandis (GG100) e de sementes híbridas de Eucalyptus urophylla x Eucalyptus grandis. O estudo foi realizado no sul do estado do Tocantins, em área da empresa PROJECTO no município de Aliança (TO). Os dados foram obtidos a partir de inventário estratificado (por híbrido) contínuo, com parcelas retangulares de 27,5 x 28 $\mathrm{m}\left(770 \mathrm{~m}^{2}\right)$ cada, distribuídas de forma sistemática em cada estrato. As variáveis dendrométricas avaliadas foram o CAP, em cm (após convertido em DAP) e Altura total (Ht), em m. Os resultados obtidos com base no ganho em altura das árvores ao longo das duas idades avaliadas evidenciaram o desempenho superior do clone GG100 em relação aos demais materiais genéticos avaliados, incremento médio de 14,4 $\mathrm{m}$ de altura aos 25 meses e $20,7 \mathrm{~m}$ aos 89 meses de idade. O incremento médio periódico para as variáveis DAP $(\mathrm{cm})$ e Ht (m) foi de 6,65 cm e 8,06 m para híbridos Eucalyptus urophylla $\times$ Eucalyptus camaldulensis (VM 58); 9,4 cm e 6,44 m para híbrido de Eucalyptus urophylla x Eucalyptus grandis (GG100) e de 9,61 cm e 8,36 m para sementes híbridas de Eucalyptus. urophylla x Eucalyptus grandis .Os híbridos de Eucalyptus urophylla $\mathrm{x}$ Eucalyptus grandis (GG100) e sementes de Eucalyptus. urophylla $\mathrm{x}$ Eucalyptus grandis obtiveram maior média de crescimento em altura total $(\mathrm{m})$ sendo estes recomendados para plantios na região. 


\section{INTRODUÇÃO}

A área total de árvores plantadas no Brasil alcançou 7,83 milhões de hectares em 2018, estável em relação ao ano de 2017, devido exclusivamente ao aumento das áreas com eucalipto. O setor brasileiro de árvores plantadas é responsável por $91 \%$ de toda a madeira produzida para fins industriais e $6,2 \%$ do PIB Industrial no País (Ibá, 2019).

O gênero Eucalyptus é o mais utilizado no Brasil para atender à demanda de madeira para celulose, serraria, energia, dentre outros usos (Almeida et al., 2004). Apresenta inúmeras qualidades, tais como, espécie de rápido crescimento, grande incremento de matéria seca, facilidade de manejo, diversidade de espécies, atende diversos ramos industriais, além da alta taxa de produção de sementes e grande aptidão na propagação vegetativa (Fernandes et al., 2012).

A produção de Eucalyptus teve grande aumento em ampla parte do país, graças ao melhoramento genético da espécie para as diversas condições ambientais brasileiras, que ligado às melhorias na área de manejo florestal, tem possibilitado a fixação de povoamentos férteis e apropriados em diferentes regiões, condizentes com os usos diferentes da madeira (Martins et al., 2005). Segundo Garber et al. (2003) com o aumento da intensidade de utilização e manejo desta espécie e a diminuição dos limites entre classes de comercialização, métodos mais precisos para estimar suas variáveis são necessários. Entretanto, requer se o uso de técnicas analíticas apropriadas para estimar a significância estatística dos resultados e respeitar a estrutura de erros dos dados. De acordo Reis et al. (1992) até poucos anos atrás, o inventário de florestas no Brasil era realizado por meio de simples levantamento do estoque de indivíduos de grande porte, susceptíveis de serem explorados, resultando numa visão incompleta e por vezes distorcida da verdadeira condição de desenvolvimento da floresta.

Os inventários tornaram se muito mais complexos e informativos, com a evolução da tecnologia e a constante pressão dos órgãos ambientais, neste novo enfoque, os inventários que na maioria dos casos eram utilizados para determinação do volume de madeira existente na floresta, passaram a ser utilizados para determinação de outros aspectos como volume total, volume comercial da floresta, a avaliação da regeneração natural das espécies, e outras peculiaridades inerentes ao objetivo do inventário florestal. O Inventário Florestal é o procedimento utilizado para obter informações sobre as características quantitativas e qualitativas da floresta e de muitas outras características das áreas sobre as quais a floresta está desenvolvendo (Bertram et al, 1982).

As informações geradas pelo inventário florestal contínuo alimentam o planejamento estratégico e influenciam diretamente as tomadas de decisão na gestão dos ativos florestais nas empresas do setor florestal brasileiro (Guedes et al., 2012).

Segundo Martins (2015), o DAP é uma variável de fácil obtenção, oferecendo correlação alta com o volume e altura, essa variável se configura como um dado independente e essencial para a construção de modelos matemáticos utilizados na mensuração florestal quando aplicados em regressões. Já a altura é uma variável dendrométrica que pode ser utilizada para indicar a qualidade de um local e comprovar a adaptabilidade de uma espécie no mesmo (Santos et al. 2015). Segundo Campos e Leite (2006) este parâmetro avalia a capacidade produtiva de um local, que é o "potencial para produção de madeira (ou outro produto) de um determinado lugar, para determinada espécie ou clone".

Contudo, a altura total e o diâmetro à altura do peito (DAP), são os dois principais parâmetros utilizados para o cálculo da área basal e do volume que existe em uma floresta. (Viera et al., 2014).

A hipótese aqui testada é de que independente do híbrido de Eucalyptus avaliado o crescimento e incremento em altura é o mesmo. Desta forma e tendo vista a necessidade de se conhecer acerca do desempenho e o comportamento silvicultural dos materiais genéticos que compõem os plantios no Tocantins, este trabalho objetivou avaliar o desenvolvimento em altura de materiais híbridos de $E u$ calyptus urophylla x Eucalyptus camaldulensis, Eucalyptus urophylla $\mathrm{x}$ Eucalyptus grandis e de sementes hibridas de Eucalyptus urophylla x Eucalyptus grandis no município de Aliança- TO.

\section{MATERIAIS E MÉTODOS}

\section{Caracterização da área de estudo}

O presente estudo foi realizado em área de plantio comercial de Eucalyptus spp. localizado no sul do Estado do Tocantins, em propriedade privada na zona rural do município de Aliança $\left(71^{\circ} 23^{\prime} 71^{\prime \prime}\right.$ e $87^{\circ} 44^{\prime} 10^{\prime \prime} \mathrm{S}$ ) distante $167 \mathrm{~km}$ da capital Palmas TO. Possui elevação de $280 \mathrm{~m}$ de altitude.

Segundo classificação climática de Köppen, o clima predominante na região é tropical sub úmido com moderada deficiência hídrica, sendo classificado com Aw. A precipitação anual média é de $1500 \mathrm{~mm}$, com período chuvoso estende-se de outubro a abril e o período de estiagem entre os meses de maio a setembro. A temperatura média varia entre mínima de $25^{\circ}$ e máxima de $30^{\circ} \mathrm{C}$.

Quanto ao manejo da área foi realizada uma adubação de base incorporada 3 meses antes do plantio feito com subsolador, aplicando-se o fosfato natural 
no fundo do sulco. Após foram realizadas duas Adubações de cobertura: a primeira cobertura foi realizada aos 90 dias após o plantio e a segunda foi realizada nove meses após o plantio. Após esse período foi realizada uma adubação de manutenção com 24 meses após o plantio.

No povoamento foram realizados tratos culturais como: limpeza mecanizada, combate à formiga e cupim. Foi realizado um replantio num período de 30 dias após o plantio. O povoamento não recebeu nenhum tipo de tratos silviculturais sendo que a desrama natural ocorre naturalmente em florestas de eucalipto, sendo que nenhuma medida especial deve ser tomada a fim de promovê-la.

O tipo de solo é classificado como sendo Latossolo Vermelho Amarelo e textura média com predomínio da fração areia.

A análise química de solo feita nos dois períodos (Tabela 1) Segundo a Comissão de Química e Fertilidade do Solo - RS/SC (2004), o teor de matéria orgânica no solo é baixo ( menor que $\leq 2,5 \%$ ); o pH é ácido ( valor < 7), o P disponível é muito baixo ( valores menores que $\leq 3,0 \mathrm{e} \leq 5,0 \mathrm{mg} \mathrm{dm}^{-3}$, respectivamente); o K trocável é baixo ( valores menores que $40-120 \mathrm{mg} \mathrm{dm}^{-3}$ ); o Ca e $\mathrm{Mg}$ trocáveis são baixos ( valores menores que o intervalo de $\leq$ $2,0 \mathrm{a} \leq 0,5 \mathrm{cmol}_{\mathrm{c}} \mathrm{dm}^{-3}$, respectivamente); a saturação por $\mathrm{Al}$ é alta (> 20\%) e a saturação por bases é de baixa a média ( valores menores que o intervalo de $\leq 20$ a $<45 \%$ ). Com isso, de maneira geral, o solo da área experimental é de baixa fertilidade.

Tabela 1 - Análise química e física do solo do talhão I aos 25 e 89 para os híbridos Eucalyptus urophylla x Eucalyptus camaldulensis (VM 58), Eucalyptus urophylla $\mathrm{x}$ Eucalyptus grandis (GG100) e de sementes híbridas de Eucalyptus. urophylla x Eucalyptus grandis no município de Aliança - TO.

\begin{tabular}{|c|c|c|c|c|c|c|c|c|c|c|c|}
\hline \multirow{2}{*}{$\begin{array}{l}\text { Idade } \\
\text { Meses }\end{array}$} & \multicolumn{9}{|c|}{$\mathrm{cmol} / \mathrm{dm}^{3}$} & \multicolumn{2}{|c|}{$\mathrm{mg} / \mathrm{dm}^{3}(\mathrm{ppm})$} \\
\hline & $\mathrm{Ca}+\mathrm{Mg}$ & $\mathrm{Ca}$ & $\mathrm{Mg}$ & $\mathrm{Al}$ & $\mathrm{H}+\mathrm{Al}$ & $\mathrm{K}$ & CTC (T) & SB & CTC $(\mathrm{t})$ & $\mathrm{K}$ & $\mathrm{P}(\mathrm{Mel})$ \\
\hline 25 & 1,20 & 0,41 & 0,40 & 0,31 & 1,70 & 0,02 & 1,97 & 0,8 & 1,17 & 7,17 & 3,6 \\
\hline \multirow[t]{3}{*}{89} & 1,09 & 0,45 & 0,64 & 0,26 & 2,46 & 0,01 & 3,57 & 1,1 & 1,38 & 6,91 & 1,86 \\
\hline & \multicolumn{2}{|c|}{$(\%)$} & \multicolumn{2}{|c|}{ Mat. Orgânica } & \multicolumn{3}{|c|}{$\mathrm{pH}$} & \multicolumn{4}{|c|}{ Textura (\%) } \\
\hline & $\mathrm{V}$ & $\mathrm{M}$ & $\%$ & $\mathrm{~g} / \mathrm{dm}^{3}$ & $\mathrm{CaCl}_{2}$ & $\mathrm{H}_{2} \mathrm{O}$ & $\mathrm{KCl}$ & Areia & $\mathrm{Sil}$ & & Argila \\
\hline 25 & 35,05 & 31,02 & 0,51 & 5,14 & 4,02 & 5,01 & - & 73,03 & 5, & & 21,23 \\
\hline 89 & 33,39 & 19,33 & 0,51 & 5,11 & 4,47 & 5,14 & & 58,32 & 7,6 & & 33,98 \\
\hline
\end{tabular}

Onde: $\mathrm{V}(\%)=100 * \mathrm{SB} \div \mathrm{CTC} ; \mathrm{SB}=\mathrm{Ca}^{2+}+\mathrm{Mg}^{+}+\mathrm{K}^{+} ; \mathrm{CTC}=\mathrm{Ca} 2++\mathrm{Mg} 2++\mathrm{K}++\mathrm{H}++\mathrm{Al}+3$.

Fonte: Laudo do Laboratório de Análises de Solos da Universidade Federal do Tocantins - Campus de Gurupi, (2011 e 2016).

\section{Base de Dados}

A base de dados utilizada foi composta por dados dendrométricos nos anos de 2011 (25 meses de idade) e 2016 ( 89 meses de idade), dos híbridos Eucalyptus urophylla x Eucalyptus camaldulensis (VM 58), Eucalyptus urophylla x Eucalyptus grandis (GG100), ambos plantados por estacas e de sementes híbridas de Eucalyptus. urophylla x Eucalyptus grandis provenientes de uma área total correspondente a 109, 14 ha plantados em abril de 2009 (com 4 meses de viveragem) sendo as mudas obtidas em viveiro na própria região.

Os dados foram obtidos a partir do inventário florestal contínuo, com parcelas permanentes, sendo a forma das unidades amostrais retangulares de dimensões $27,5 \times 28 \mathrm{~m}\left(770 \mathrm{~m}^{2}\right.$ com espaçamento entre árvores de 2,5 x $4 \mathrm{~m}$ ) cada situadas em linhas equidistantes $125 \mathrm{~m}$ uma das outras e $80 \mathrm{~m}$ entre parcelas distribuídas sistematicamente e estratificadas num total de 42 parcelas (14 parcelas por estrato) do inventário piloto, onde a intensidade amostral foi determinada com 33,47 parcelas.

A intensidade amostral foi determinada com base no DAP a partir do inventário piloto pela seguinte equação:

$$
\mathrm{n}=\frac{\mathrm{t}_{\infty / 2}^{2} \cdot(\mathrm{CV})^{2}}{(\mathrm{E} \%)^{2}}
$$

Onde: $\mathrm{n}=$ quantidade de unidades amostrais; $\mathrm{t} \propto / 2=$ valor tabelado da distribuição t de Student ( $\alpha 5 \%$, $\mathrm{n}-1 \mathrm{gl}) ; \mathrm{CV}=$ coeficiente de variação, em percentagem, e E\% = erro de amostragem.

A estratificação foi determinada de acordo com os híbridos em três estratos com tamanhos de 47,93 ha para os híbridos de Eucalyptus urophylla x Eucalyptus camaldulensis (VM 58); 36,65 ha para Eucalyptus urophylla $\mathrm{x}$ Eucalyptus grandis (GG100) e 24,56 ha para sementes híbridas de Eucalyptus urophylla $\mathrm{x}$ Eucalyptus grandis.

As variáveis dendrométricas avaliadas foram: altura total das sete primeiras (medida em metros com clinômetro e as demais foram determinadas por relação hipsométrica) e CAP (circunferência à altura do peito a $1,30 \mathrm{~m}$ do solo, medida em centímetros com fita métrica e após convertido em 
DAP).

Foi utilizado o modelo polinômio de terceiro grau ajustado por Campos (1986) para cálculo das demais alturas totais. Sendo na primeira descrita pelo modelo: $\quad \operatorname{lnh}=2,02733-4,4891$ * $(1 / D A P)-0,40595 * \ln (D A P)$ com $\mathrm{R}^{2} \mathrm{aj}=0$, 786 e Syx\% $=9,87$.

$\mathrm{O}$ incremento periódico e o médio foram calculados com base nas equações descritas por Schneider, 1993, sendo:

$$
I P=X_{n+1}-X_{n} \quad I M A=X_{i} / i
$$

Em que: $X_{n}=$ variável dendrométrica no início do período; $X_{n+1}=$ variável dendrométrica no final do período, $X_{i}=$ variável na idade $i ; i=$ idade do povoamento.

As análises estatísticas foram feitas com o uso dos softwares ${ }^{\circledR}$ Minitab versão 18 , ${ }^{\circledR}$ Sigmaplot versão 12.0 e ${ }^{\circledR E x c e l . ~}$

\section{RESULTADOS E DISCUSSÃO}

Durante os períodos levantados (25 e 89 meses) para os híbridos Eucalyptus urophylla $\mathrm{x}$ Eucalyptus camaldulensis (VM 58), Eucalyptus urophylla $\mathrm{x}$ Eucalyptus grandis (GG100) e sementes híbridas de Eucalyptus urophylla x Eucalyptus grandis A dispersão dos dados ao longo do período de tempo avaliado é considerada baixa $(\mathrm{CV} \%<20)$, indicando baixa variação nos dados com exceção do Sementes de GG100 aos 25 meses que é considerada média (Gomes, 1990).

Para variável dendrométrica $\mathrm{Ht}(\mathrm{m})$ com casca nos períodos levantados ( 25 e 89 meses) para os híbridos Eucalyptus urophylla x Eucalyptus camaldulensis (VM 58), Eucalyptus urophylla x Eucalyptus grandis (GG100) e de sementes de Eucalyptus urophylla $\mathrm{x}$ Eucalyptus grandis a dispersão dos dados ao longo do período de tempo avaliado é considerada baixa (CV\% < 20), indicando baixa variação nos dados (Gomes, 1990). Tanto para a variável DAP como Ht o híbrido Eucalyptus urophylla $\mathrm{x}$ Eucalyptus grandis (GG100) foi o que obteve maior incremento nos dois períodos de temo avaliados.

Valores inferiores foram encontrados por Ferreira et al. (2014) em plantios de Eucaliptus grandis com espaçamento de $3 \times 1 \times 2 \mathrm{~m}$ para variável DAP (cm) sendo valor médio de $8,3 \mathrm{~cm}$ aos 25 meses de idade em Botucatu (SP). Também Ferreira et al (2014) encontraram valores inferiores a estes quando avaliaram o crescimento para mudas de $E u$ calyptus urophylla $\mathrm{x}$ Eucalyptus. grandis aos seis anos encontrando valores médios de DAP de 15,6 $\mathrm{cm}$ em Avaré (SP) em espaçamento 3 x $2 \mathrm{~m}$. Fato este justificado pelo espaçamento que influencia de forma direta na taxa de crescimento.

A dispersão dos dados da HT ao longo do período de tempo avaliado é considerada baixa (CV\% <20), indicando baixa variação nos dados de HT da população (Gomes, 1990). Estes resultados demonstram uma heterogeneidade entre parcelas no que se refere ao DAP e Ht de fato que pode ser explicado pela influência dos materiais genéticos ou devido a problemas de instalação no plantio do povoamento. Os valores podem ser plenamente aceitos para interpretar a abrangência da amostragem (Tabela 2).

Tabela 2 - Estatística descritiva para DAP (cm) e Ht (m) para os híbridos Eucalyptus urophylla x Eucalyptus camaldulensis (VM 58), híbrido de Eucalyptus urophylla x Eucalyptus grandis (GG100) e de sementes de Eucalyptus urophylla x Eucalyptus grandis aos 25 e 89 meses de idade em Aliança (TO).

\begin{tabular}{lccc|ccc}
\hline \multirow{2}{*}{ Estatísticas } & \multicolumn{3}{c|}{25 meses } & \multicolumn{3}{c}{89 meses } \\
\cline { 2 - 7 } & Híbrido & Híbrido & Seminal & Híbrido & Híbrido & Seminal \\
VM 58 & GG100 & GG100 & VM 58 & GG100 & GG100 \\
\hline DAP mínimo (cm) & 7,36 & 1,59 & 2,28 & 10,1 & 14,2 & 14 \\
DAP máximo (cm) & 12,41 & 13,05 & 14,32 & 24,6 & 25,4 & 21,9 \\
Média DAP (cm) & 10,25 & 10,40 & 8,69 & 16,9 & 19,8 & 18,3 \\
DP DAP (cm) & 1,44 & 1,31 & 2,11 & 1,27 & 2,11 & 2,24 \\
CV\% DAP & 14,74 & 12,67 & 24,35 & 9,11 & 12,67 & 15,31 \\
N de observações & 913 & 684 & 454 & 1634 & 487 & 839 \\
Ht mínima (m) & 6,2 & 9,1 & 7 & 11 & 14 & 15 \\
Ht máxima (m) & 14,5 & 16,4 & 16 & 24 & 26,5 & 22 \\
Ht média (m) & 10,44 & 14,16 & 10,84 & 18,5 & 20,6 & 19,2 \\
DP Ht (m) & 0,80 & 0,93 & 0,48 & 2,08 & 2,00 & 1,42 \\
CV\% Ht(m) & 7,72 & 6,62 & 4,45 & 10,82 & 10,1 & 7,4 \\
N de observações & 120 & 98 & 59 & 202 & 47 & 89 \\
\hline
\end{tabular}

O incremento médio periódico para as variáveis

DAP $(\mathrm{cm})$ e $\mathrm{Ht}(\mathrm{m})$ foi de $6,65 \mathrm{~cm}$ e $8,06 \mathrm{~m}$ para 
híbridos Eucalyptus urophylla x Eucalyptus camaldulensis (VM 58); 9,4 cm e 6,44 m para híbrido de Eucalyptus urophylla $\mathrm{x}$ Eucalyptus grandis (GG100) e de 9,61 cm e 8,36 m para sementes híbridas de Eucalyptus. urophylla x Eucalyptus grandis .

Já a taxa de incremento médio anual em DAP e altura total aos 25 meses para Eucalyptus urophylla x Eucalyptus grandis (GG100) 5,4 cm e 6,80 m, Eucalyptus urophylla $x$ Eucalyptus camaldulensis (VM 58) sendo 5,1 cm e 5,22 m e sementes de Eucalyptus. urophylla x Eucalyptus grandis com 4,3 $\mathrm{cm}$ e 7,5 m e para os 89 meses para Eucalyptus urophylla x Eucalyptus grandis (GG100) $2,65 \mathrm{~cm}$ e 2,69 m, Eucalyptus urophylla $x$ Eucalyptus camaldulensis (VM 58) sendo 2,28 cm e 2,5 m e sementes de Eucalyptus. urophylla x Eucalyptus grandis com 2,46 cm e 2,61 m respectivamente, o que indica que a espécie apresenta crescimento condizente com os encontrados na literatura para a região de estudo, embora não haja ainda progênie adaptada especificamente.

Isso demonstra um desempenho característico da espécie com uma redução gradativa na velocidade de crescimento dessas variáveis, provocando a necessidade de intervenções na densidade do povoamento ao longo da rotação a fim de evitar a estagnação do crescimento. Além disso, a intervenção na densidade, estimula maior crescimento e produção das árvores, dependendo da intensidade do desbaste e fatores vinculados ao sítio (Damásio et al. 2015).

Silva (2016) ao avaliar encontrou para plantios clonais híbridos não desbastados de Eucalyptus urophylla $x$ Eucalyptus grandis no estado do Pará valores de 4,04 $\mathrm{cm}$ e 8,49 $\mathrm{m}$ aos 24 meses de idade. Já aos 72 meses os valores encontrados foram de $27,12 \mathrm{~cm}$ e $32,68 \mathrm{~m}$.

Santos et al. (2012) encontraram valores do IMA em altura total de 3,408 m para o clone de GG100 em Minas Gerais e na região do Médio Parnaíba (RJ), Ferreira et al. (2017) encontraram valor de incremento periódico (18 para 80 meses) para $\mathrm{Eu}$ calyptus grandis o valor de $18 \mathrm{~m}$ de $\mathrm{Ht}$ e de 10,4 $\mathrm{cm}$ de DAP.

Ferreira et al. (2014), observou um crescimento em altura de 23,1 m em clones de GG100 implantados em espaçamento de $3 \times 2,5$ no município de Avaré - SP, aos 5 anos de idade, valor esse, próximo aos do clone GG100 avaliados nesse experimento aos 7 anos de idade. Enquanto que Tonini et al. (2006), observou um crescimento em altura de clones urograndis na região de Cantá em Roraima, entre 15,1 a 21,6 m, aos 6 anos de idade. Oliveira et al. (2009), realizou um experimento no Noroeste de Minas Gerais com clones de VM58, e verificou um crescimento em altura, 51 meses após o plantio, de 19,27 e $20,59 \mathrm{~m}$ para os espaçamentos $3,33 \times 2$ $\mathrm{m}$ e $3,33 \times 3 \mathrm{~m}$ respectivamente Vale et al. (2012) híbrido E. urograndis GG-100 com procedência de Dom Aquino - MT e idade de 36 meses encontrou diâmetro médio de $13,93 \mathrm{~cm}$ e média da altura total encontrada foi de $18,40 \mathrm{~m}$.

Miranda et al. (2019) ao estudarem a produtividade de híbridos de Eucalyptus no Mato Grosso em espaçamento de $3 \times 3 \mathrm{~m}$, aos cinco anos de idade concluíram que o Eucalyptus urophylla $\mathrm{x}$ Eucalyptus grandis (GG100) obteve valores médios de DAP de 14,22 cm. Estes resultados confirmam a grande adaptabilidade destes materiais às diversas condições de solo e clima.

A maior frequência relativa de indivíduos por classe para a variável $\mathrm{Ht}(\mathrm{m})$ para o híbrido $E u$ calyptus urophylla $x$ Eucalyptus camaldulensis (VM 58) foi de $45 \%$ no intervalo de 12 a $14 \mathrm{~m}$ aos 25 meses e de $43,56 \%$ aos 89 meses no intervalo de 18;20 m. Para o híbrido de Eucalyptus urophylla x Eucalyptus grandis (GG100) foi de $66,32 \%$ no intervalo de 14 a $16 \mathrm{~m}$ aos 25 meses e de $51,06 \%$ no intervalo de 16 a $20 \mathrm{~m}$ aos 89 meses e para sementes de Eucalyptus. urophylla $x$ Eucalyptus grandis foi de $81,35 \%$ no intervalo de 9 a 12 aos 25 meses e de $65,16 \%$ no intervalo de 17 a $20 \mathrm{~m}$ aos 89 meses. Observou-se que de acordo com a distribuição de frequência por classes de altura total (Figura 01) que a medida que o tempo passa existe recrutamento das árvores de menores alturas para as de maiores caracterizando assim que a distribuição de frequência dos indivíduos não é constante com o tempo caracterizando uma distribuição normal.

A interação do genótipo com o ambiente é uma das características mais importantes na recomendação ou escolha de cultivares florestais, muito embora na atualidade existam genótipos que são classificados como plásticos, por apresentarem bom desenvolvimento em uma multiplicidade de ambientes, como é o caso do Eucalyptus urophylla x Eucalyptus grandis (GG100) (Filho e Santos, 2013) demostrado pelos resultados que indicam uma maior frequência de indivíduos nas maiores classes de altura nos dois períodos de tempo avaliados.

A uniformidade dos plantios dos clones avaliados (Figura 1), é uma característica marcante da silvicultura clonal em plantações de eucalipto, o que possibilita maior controle sobre a qualidade dos produtos; maximização em ganho em produtividade silvicultural; aumenta a eficiência nos tratos sulviculturais de poda e desbastes; além de facilitar as operações mecanizáveis de adubação e controle 
plantas espontâneas, pragas e doenças (Xavier et al., 2013).

Foi aplicado o teste de normalidade de Kolmogorov-Smirnov aos 25 e aos 89 meses para cada procedência, hipótese de que os dados referentes à mesma tenham uma distribuição normal (Figura 2).

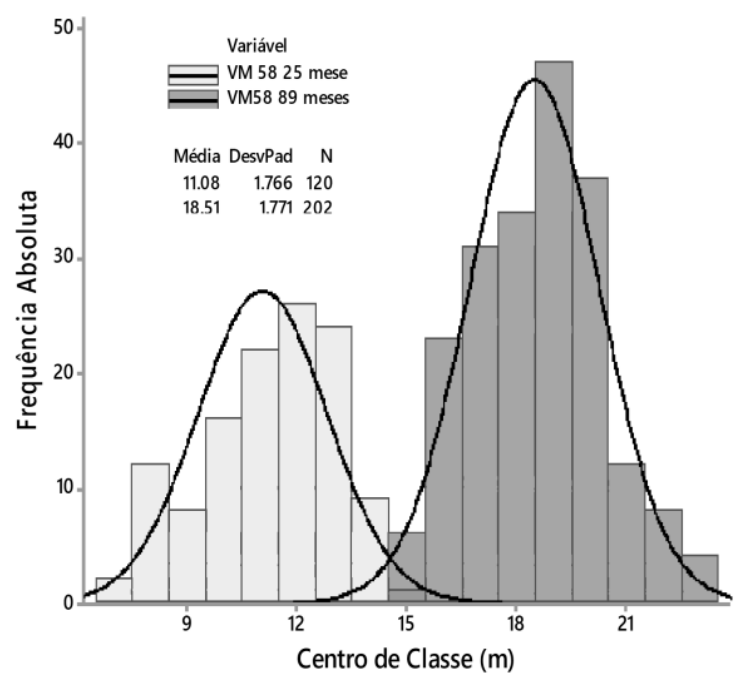

(a)

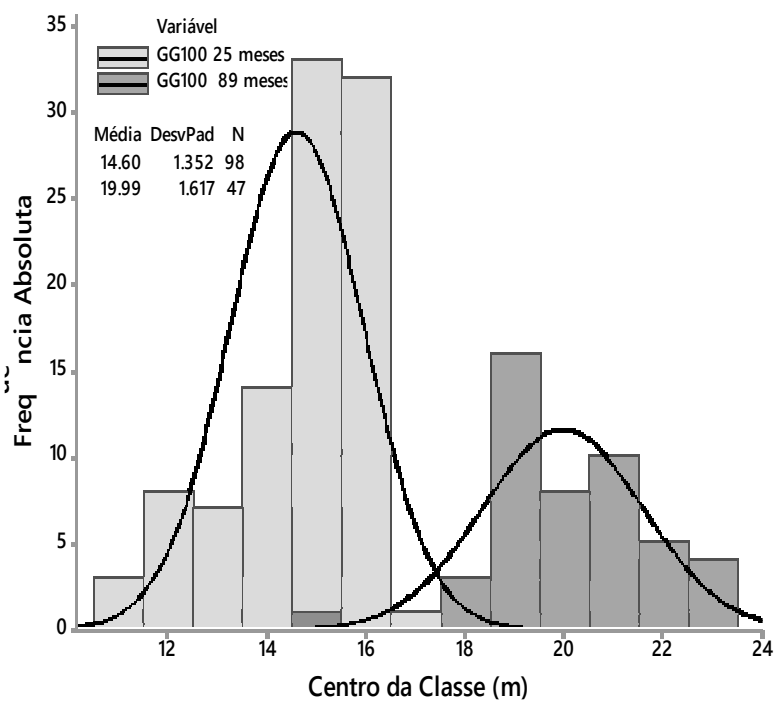

(b)

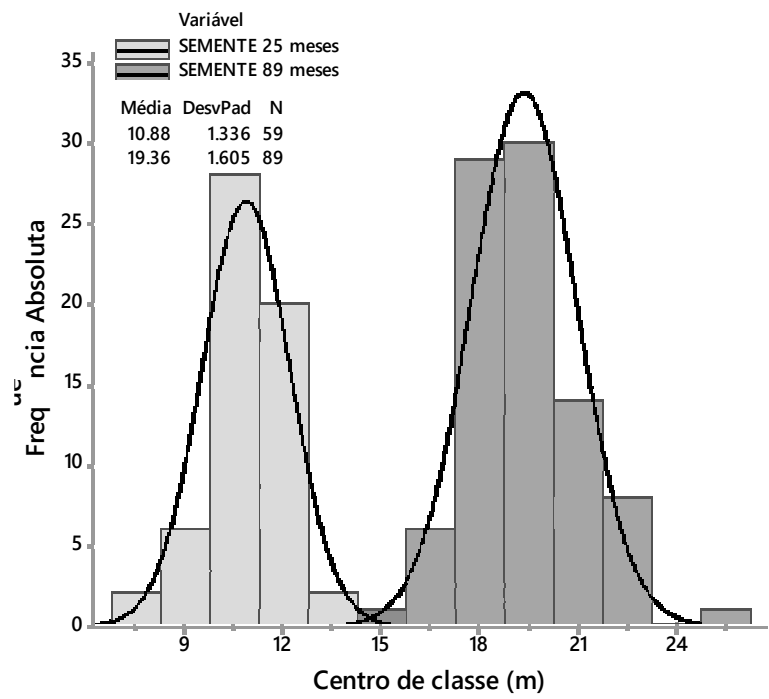

(c)

Figura 01 - Frequência absoluta para variável Ht (m) aos 25e 89 meses para os híbridos de Eucalyptus urophylla x Eucalyptus camaldulensis (VM 58) (a), híbrido de Eucalyptus urophylla x Eucalyptus grandis (GG100) (b) e de sementes de Eucalyptus. urophylla x Eucalyptus grandis (c).

Aos 25 meses a normalidade foi comprovada para híbrido de Eucalyptus urophylla $x$ Eucalyptus camaldulensis (VM 58) e semente de híbrido de Eucalyptus urophylla $x$ Eucalyptus grandis. Já aos 89 meses todos os híbridos se caracterizaram como uma distribuição normal, sendo considerando $\alpha=$ 0,05 e $\mathrm{n}=202$ para o híbrido de Eucalyptus urophylla $x$ Eucalyptus camaldulensis (VM 58), considerando o valor de $\mathrm{D}_{\mathrm{n}} 0,072<0,095$; para híbrido de Eucalyptus urophylla $x$ Eucalyptus grandis (GG100) considerando $\alpha=0,05$ e $\mathrm{n}=89$ o valor de $\mathrm{D}_{\mathrm{n}} 0,133<0,198$ e para semente de híbrido de Eucalyptus urophylla $x$ Eucalyptus grandis o valor de $\mathrm{D}_{\mathrm{n}} 0,096<0,144$. 

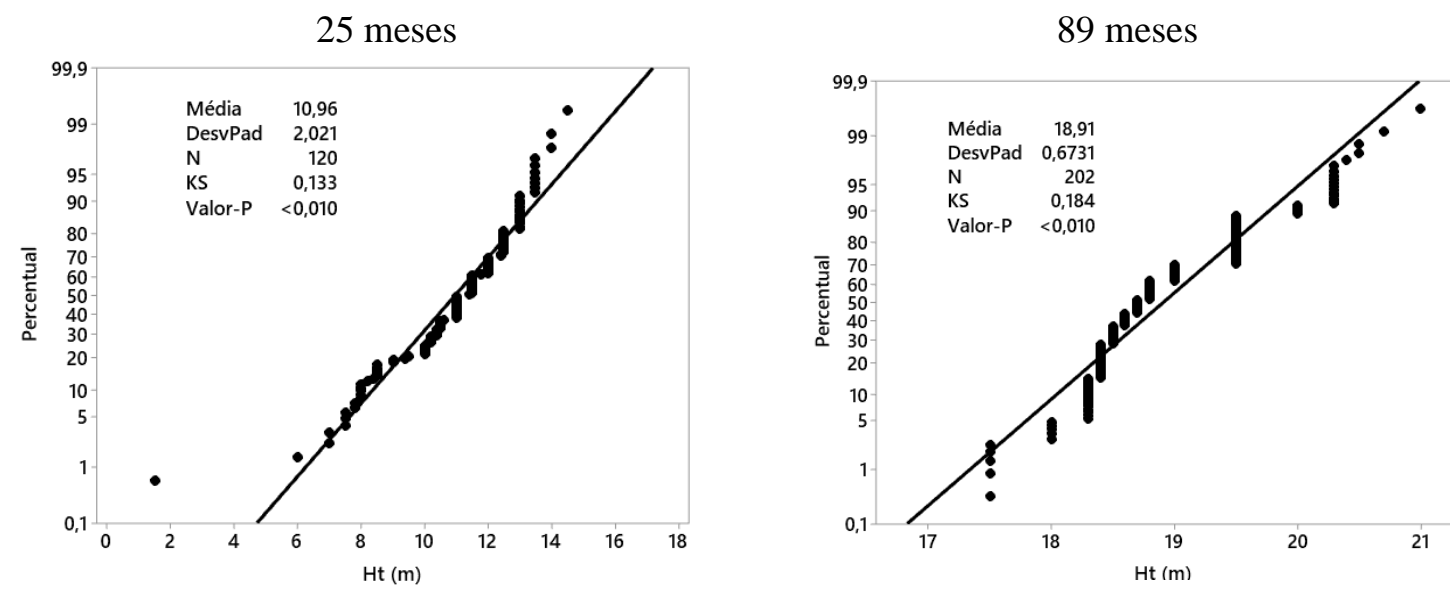

( a )
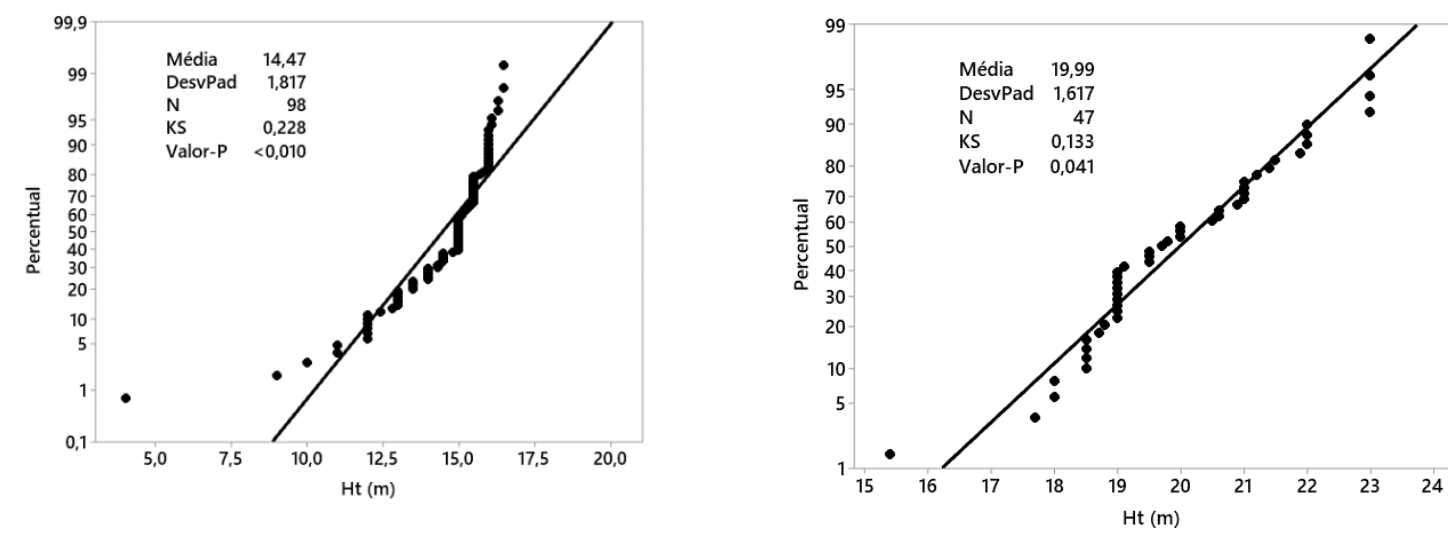

( b )
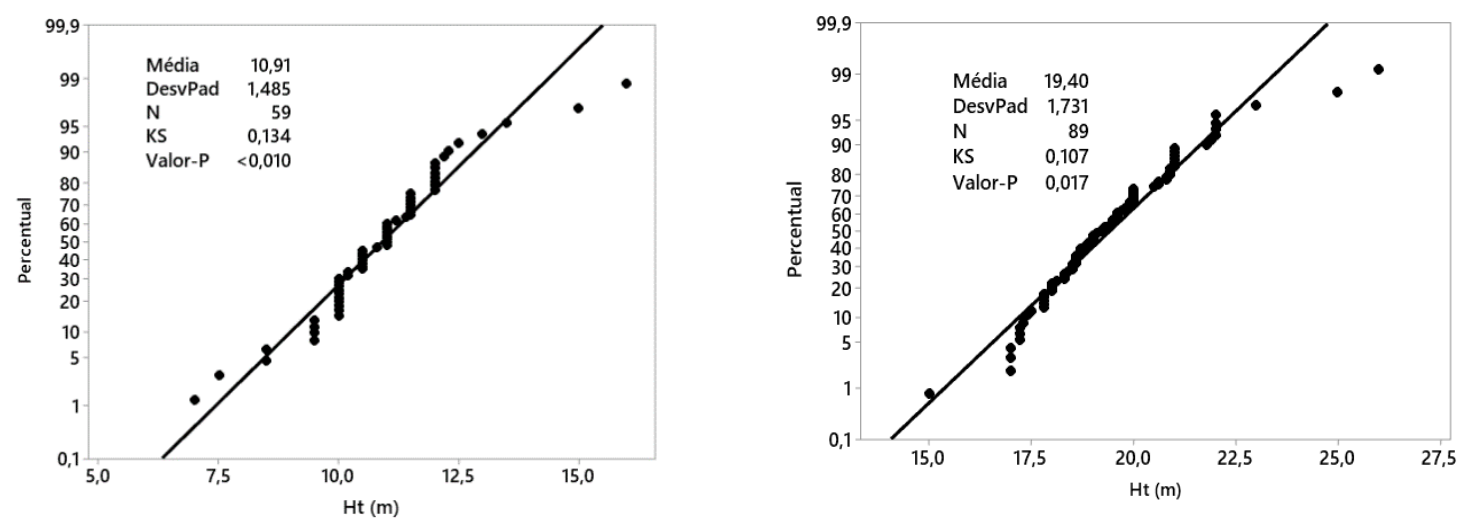

(c)

Figura 2 - Teste de normalidade Kolmogorov-Smirnov para os híbridos de Eucalyptus urophylla x Eucalyptus camaldulensis (VM 58) (a), híbrido de Eucalyptus urophylla x Eucalyptus grandis (GG100) (b) e semente de híbrido de Eucalyptus urophylla x Eucalyptus grandis (c).

De acordo com a análise de variância (Tabela 3), tanto aos 25 meses como aos 89 , pode-se concluir que existe pelo menos dois híbridos diferem significativamente em altura total ao avaliar o valor- $\mathrm{p}=$
0,000 (menor que o nível de significância estabelecido de 0,05$)$ e por ter um $\mathrm{F}$ significativo. 
Tabela 3 - Análise de variância para os híbridos Eucalyptus urophylla x Eucalyptus camaldulensis (VM 58), híbrido de Eucalyptus urophylla x Eucalyptus grandis (GG100) e de sementes de Eucalyptus urophylla x Eucalyptus grandis.

\begin{tabular}{|c|c|c|c|c|c|c|c|c|}
\hline \multicolumn{9}{|c|}{25 meses } \\
\hline Fonte & GL & SQ Seq. & Contribuição & SQ (Aj.) & $\mathrm{QM}(\mathrm{Aj})$. & & Valor F & Valor-P \\
\hline Fator & 2 & 818.6 & $55.67 \%$ & 818.6 & \multicolumn{2}{|c|}{409.293} & $172.03^{*}$ & 0.000 \\
\hline Erro & 274 & 651.9 & $44.33 \%$ & 651.9 & \multirow{2}{*}{\multicolumn{2}{|c|}{2.379}} & & \\
\hline Total & 276 & 1470.5 & $100.00 \%$ & & & & & \\
\hline \multicolumn{9}{|c|}{89 meses } \\
\hline Fonte & GL & SQ Seq & Contribuição & SQ (Aj.) & $\mathrm{QM}(\mathrm{Aj})$. & Valor F & Valor-P & \\
\hline Fator & 2 & 104.4 & $9.65 \%$ & 104.4 & 52.206 & $17.90 *$ & 0.000 & \\
\hline Erro & 335 & 977.1 & $90.35 \%$ & 977.1 & 2.917 & & & \\
\hline Total & 337 & 1081.5 & $100.00 \%$ & & & & & \\
\hline
\end{tabular}

No teste de Tukey (Tabela 4) com nível de significância de $5 \%$ observamos que tanto aos 25 meses como aos 89 meses existe diferença significativa entre as alturas totais dos diferentes híbridos avaliados, sendo que são iguais entre si os híbridos de Eucalyptus urophylla $x$ Eucalyptus camaldulensis (VM 58) e sementes de Eucalyptus urophylla $x$ Eucalyptus grandis aos 25 meses e os híbridos de Eucalyptus urophylla $x$ Eucalyptus grandis (GG100) e sementes de Eucalyptus urophylla $x$ Eucalyptus grandis aos 89 meses.

Tabela 4 - Teste de Tukey para os híbridos avaliados.

\begin{tabular}{|c|c|c|c|c|}
\hline & Variáveis & $\mathrm{N}$ & $\begin{array}{l}\text { Média } \\
\mathrm{Ht}(\mathrm{m})\end{array}$ & Tukey \\
\hline \multirow{3}{*}{25 meses } & Eucalyptus urophylla $x$ Eucalyptus grandis (GG100) & 98 & 14.60 & $\mathrm{a}$ \\
\hline & Eucalyptus urophylla $x$ Eucalyptus camaldulensis (VM 58) & 120 & 11.07 & $\mathrm{~b}$ \\
\hline & Sementes de Eucalyptus. urophylla x Eucalyptus grandis & 59 & 10.87 & $\mathrm{~b}$ \\
\hline \multirow{3}{*}{89 meses } & Eucalyptus urophylla $x$ Eucalyptus grandis (GG100) & 47 & 19.98 & $\mathrm{a}$ \\
\hline & Sementes de Eucalyptus. urophylla x Eucalyptus grandis & 89 & 19.35 & a \\
\hline & Eucalyptus urophylla x Eucalyptus camaldulensis (VM 58) & 202 & 18.50 & $\mathrm{~b}$ \\
\hline
\end{tabular}

Obs: Médias que não compartilham uma letra são significativamente diferentes.

O híbrido de Eucalyptus urophylla x Eucalyptus grandis (GG100), diferiu significativamente ao nível de 5\% dos demais híbridos, tendo um crescimento em altura de $25 \%$ a mais que os outros híbridos na idade de 25 meses e de 3,15\% e 7,40\% para Sementes de Eucalyptus. urophylla x Eucalyptus grandis e Eucalyptus urophylla $x$ Eucalyptus camaldulensis (VM 58) respectivamente aos 89 meses.

Os resultados deste levantamento indicam que o tipo de material genético bem como suas adaptações edafoclimáticas, tipo de manejo inicial, e capacidade de resistência à competição interespecífica são fatores decisivos para a produtividade das plantas cultivadas.

\section{CONCLUSÕES}

O híbrido de Eucalyptus urophylla x Eucalyptus grandis (GG100) obteve os melhores resultados para os parâmetros DAP (cm), Ht (m), IMA em altura total e IP em altura total nos dois períodos de tempo avaliados.

Para ambos os períodos avaliados (25 e 89 meses) os híbridos de Eucalyptus urophylla x Eucalyptus grandis (GG100) e sementes de Eucalyptus urophylla $\mathrm{x}$ Eucalyptus grandis obtiveram maior média de crescimento em altura total $(\mathrm{m})$ possuindo uma maior capacidade adaptativa, sendo estes recomendados para plantios na região.

\section{REFERÊNCIAS BIBLIOGRÁFICAS}

Almeida AC, Landsberg JJ, Sands PJ. Parameterisation of 3PG model fast-growing Eucalyptus grandis plantations. Forest Ecology and Management, Amsterdam, v.193, n.12, p.179-195, 2004.

https://doi.org/10.5902/1980509831580.

Bertram H, Charles IM, Thomas WB. Forest mensuration. 3th ed. New York: John Wiley e Sons, 1982. 401 p.

Campos ALAS. Desenvolvimento de um sistema compatível de crescimento e produção para manejo de Eucalyptus grandis (W. Hill ex-Maiden). 1986. 73f. Dissertação (Mestrado em Ciência Florestal) - Universidade Federal de Viçosa, Viçosa, 1986. 
Campos JCC, Leite HG. Mensuração florestal: perguntas e respostas. ED. UFV, $2^{\circ}$ ed., 470p., 2006.

Damásio M, da Silva FR, Santos AFA, Neto RMR. Desbaste seletivo em um povoamento de Tectona grandis implantado em sistema de integração-lavoura- pecuária-floresta. Biodiversidade, v.14, n.3, p.74, 2015.

Fernandes ALT, Florêncio TM, Faria MF. Análise biométrica de florestas irrigadas de eucalipto nos cinco anos iniciais de desenvolvimento. Revista Brasileira de Engenharia Agrícola e Ambiental, v.16, n.5, p.505-513, 2012. https://doi.org/10.1590/S1415-43662012000500006.

Ferreira ML, Silva JL, Pereira EE, Lamano-Ferreira APN. Litter fall production and decomposition in a fragment of secondary Atlantic Forest of São Paulo, SP, Southeastern Brazil. Revista Árvore, v.38, n.4, p.591-600, 2014. http://dx.doi.org/10.1590/S0100- 67622014000400002

Ferreira DHAA, Leles PS dos S, Machado EC, Abreu AHM, Ferreira FMA. Crescimento de clones de Eucalyptus urophylla $\mathrm{x}$ E. grandis em diferentes espaçamentos. Floresta, v.44, n.3, p.431-440, 2014.

http://dx.doi.org/10.5380/rf.v44i3.32188

Gomes P. Curso de estatística experimental. USP/ESALQ, Piracicaba. 1990. 468p.

Guedes IC de L, Mello JM de, Mello CR de, Oliveira AD de, Silva ST da, Scolforo JRS. Técnicas geoestatísticas e interpoladores espaciais na estratificação de povoamentos de Eucalyptus sp. Ciência Florestal, v.22, n.3, p.541-550, 2012. https://doi.org/10.5902/198050986621.

Halley D, Ferreira AA, Leles PS dos S, Neto SNO de, Paula TF, Coutinho RP, Silva RL da. Crescimento e Produção de Eucalipto na Região do Médio Paraíba do Sul. Floresta e Ambiente, v.24, p.1-9, 2017. http://dx.doi.org/10.1590/2179-8087.131315.

Ibá Indústria Brasileira De Árvores. Relatório Anual 2019. $80 \mathrm{p}$.

Martins IS, Cruz CD, Rocha M das G de B, Regazzi AJ, Pires IE. Comparação entre os processos de seleção entre e dentro e o de seleção combinada, em progênies de Eucalyptus grandis. Cerne, v.11, n.1, p.16-24, 2005.

Martins RM, Leite MVS, Cabacinha CD, Assis AL. Teste de identidade de modelos volumétricos para povoamentos de Eucalyptus sp. em sete municípios de Minas Gerais. Enciclopédia Biosfera, v.11, n.21, p.1818-1833, 2015.

Miranda DLC, Lisboa GS dos, Silva F da, Sanquetta CR, Corte APD, Condé TM. Produtividade de híbridos de eucalipto em plantios comerciais no estado de Mato Grosso. Advances in Forestry Science. v.6, n.2, p.617-621, 2019. http://dx.doi.org/10.34062/afs.v6i2.7360.

Moraes VM. Dinâmica de crescimento de eucalipto clonal sob diferentes espaçamentos, na região noroeste do estado de minas gerais. Lavras - MG - Brasil. 2006.

Oliveira TK. Desempenho silvicultural e produtivo de eucalipto sob diferentes arranjos espaciais em sistema agrossilvipastoril. Pesquisa Florestal Brasileira, Colombo, n.60, p.01-06, dez. 2009. (Edição especial). http://dx.doi.org/10.4336/2009.pfb.60.01.

Santos LC, Carvalho AMML, Pereira BLC, Oliveira AC, Carneiro ACO, Trugilho PF. Propriedades da madeira e estimativas de massa, carbono e energia de clones de $\mathrm{Eu}-$ calyptus plantados em diferentes locais. Revista Árvore, Viçosa, v.36, n.5, p.971-980, 2012. https://doi.org/10.1590/S0100-67622012000500019.

Santos AFA. Desempenho silvicultural de clones de Eucalyptus em duas regiões do estado de Mato Grosso. $2015.53 \mathrm{f}$. Dissertação (Pós-graduação em Ciências Florestais e Ambientais). Universidade Federal de Mato Grosso. Cuiabá MT.

Santos JA dos, Lima VOB, Oliveira JC de, Matos PS, Trazzi PA. Avaliação do Crescimento de Clones de eucalipto no Norte de Minas Gerais. Enciclopédia Biosfera, Centro Científico Conhecer - Goiânia, v.14, n.26, p.75, 2017. https://doi.org/10.18677/EnciBio_2017B7.

Silva MO da. Modelagem do Crescimento em Diâmetro e Altura de Árvores de eucalipto. 2016. 55p. Dissertação (Pósgraduação em Ciências Florestais). Universidade Federal de Viçosa - MG.

SEPLAN. Secretaria do Planejamento e da modernização da Gestão Pública. Atlas do Tocantins: subsídios ao planejamento da gestão territorial. 6. Ed rev. atu. Palmas: SEPLAN, 2012

Tonini H, Arco-Verde MF, Schwengber D, Junior MM. Avaliação de espécies florestais em área de mata no estado de Roraima. Cerne, v.12, n.1, p.8-18, 2006.

Valle FG, Fidelis FS, Graeff LSS, Souza LA de, Azevedo GB. Desenvolvimento inicial do híbrido Eucaliptus urograndis clone GG 100 na região de Vilhena-RO. In: 64a Reunião Anual da SBPC.2012. São Luiz - MA

Vieria M, Scumacher MV, Trüby P, Araújo EF. Biomassa e nutrientes em um povoamento de Eucalyptus urophylla $\mathrm{x}$ Eucalyptus globulus, em Eldorado do Sul-RS. Ecologia e Nutrição Florestal, v.1, n.1, p.1-13, 2013. http://dx.doi.org/10.13086/2316-980x.

Xavier A, Wedling I, Silva RL da. Silvicultura Clonal: princípios e técnicas. Viçosa, MG: Editora UFV, 2013. 279p. 